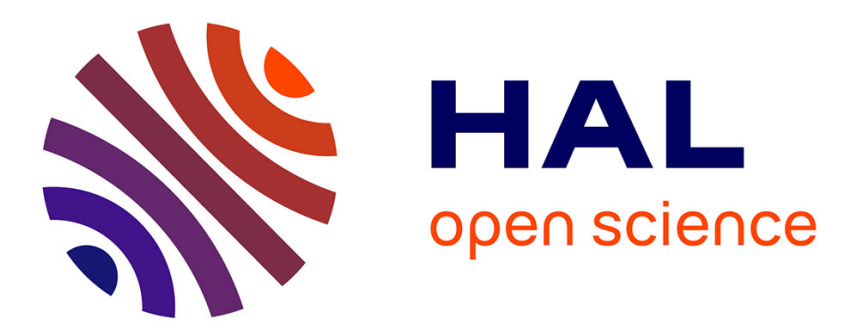

\title{
Experimental Study of electron acceleration by plasma beat-waves with $\mathrm{Nd}$ lasers
}

François Amiranoff, F. Moulin, J. Fusellier, J.M. Joly, M. Juillard, M.

Bercher, D. Bernard, A. Debraine, J.M. Dieulot, F. Jacquet, et al.

\section{- To cite this version:}

François Amiranoff, F. Moulin, J. Fusellier, J.M. Joly, M. Juillard, et al.. Experimental Study of electron acceleration by plasma beat-waves with Nd lasers. 16th IEEE Particle Accelerator Conference (PAC 95) and International Conference on High-energy Accelerators (IUPAP), May 1995, Dallas, United States. pp.634-636, 10.1109/PAC.1995.504740 . in2p3-00018994

\section{HAL Id: in2p3-00018994 https://hal.in2p3.fr/in2p3-00018994}

Submitted on 5 May 2008

HAL is a multi-disciplinary open access archive for the deposit and dissemination of scientific research documents, whether they are published or not. The documents may come from teaching and research institutions in France or abroad, or from public or private research centers.
L'archive ouverte pluridisciplinaire HAL, est destinée au dépôt et à la diffusion de documents scientifiques de niveau recherche, publiés ou non, émanant des établissements d'enseignement et de recherche français ou étrangers, des laboratoires publics ou privés. 


\title{
Experimental Study of Electron Acceleration by Plasma Beat-Waves with Nd Lasers
}

\author{
F.Amiranoff, F.Moulin, LULI Ecole Polytechnique, 91128 Palaiseau, France \\ J.Fusellier, J.M.Joly, M.Juillard, DSM-DAPNIA-SEA CEA Saclay, 91191 Gif sur Yvette, France \\ M.Bercher, D.Bernard, A.Debraine, J.M.Dieulot, F.Jacquet, P.Matricon, Ph.Miné, B.Montès, \\ R.Morano, P.Poilleux, A.Specka, LPNHE Ecole Polytechnique, 91128 Palaiseau, France \\ J.Morillo, J.Ardonceau, SESI Ecole Polytechnique, 91128 Palaiseau, France \\ B.Cros, G.Matthieussent, LPGP Université Paris Sud, 91405 Orsay, France \\ C.Stenz, GREMI Université d'Orléans, 45000 Orléans, France \\ P.Mora, CPHT Ecole Polytechnique, 91128 Palaiseau, France
}

\section{Abstract}

We have observed the acceleration of electrons by a beat-wave generated in a deuterium plasma by two Nd-YAG and Nd-YLF laser wavelengths. Electrons injected at an energy of $3.3 \mathrm{MeV}$ are observed to be accelerated up to $4.7 \mathrm{MeV}$ after the plasma. The energy gain is compatible with a peak electric field of the order of $1.2 \mathrm{GV} / \mathrm{m}$. The experiment has been performed with different injection energies, from 2.5 to $3.3 \mathrm{MeV}$, with different plasma dimensions, and with different laser intensities.

\section{Introduction}

Among new methods to accelerate particles with a large electric field, the beat-wave technique [1] has been investigated by several groups for the last decade. In this scheme two highintensity laser pulses are focused in a vessel containing hydrogen gas and create a fully ionised plasma. The beating of the two waves provides a longitudinal electric field which oscillates with the frequency difference of the two lasers. If this frequency difference is equal to the plasma frequency, a resonance effect results, and the charge separation produces a field up to several $\mathrm{GV} / \mathrm{m}$. A relativistic particle with the right phase can catch the wave and gain energy.

Two types of lasers have been considered for this purpose: the $\mathrm{CO}_{2}$ lasers at wavelengths near $10 \mu \mathrm{m}$, and the $\mathrm{Nd}$ lasers near $1 \mu \mathrm{m}$. The advantages and drawbacks of $\mathrm{CO}_{2}$ versus $\mathrm{Nd}$ lasers are briefly discussed in reference [7]. Several groups [2], [3], [4] have observed the acceleration of electrons by the first type, with an energy gain of the order of $20 \mathrm{MeV}$. We already reported [5], [6], [7] on the observation of accelerated electrons with Nd lasers in our experiment at Ecole Polytechnique. Electrons injected at an energy of $3 \mathrm{MeV}$ were observed to be accelerated up to 3.7 $\mathrm{MeV}$.

For the new data taken recently (January 1995) in the same apparatus we were able to vary several experimental parameters. In order to change the plasma dimensions we used two lenses, with different focal lengths, to focus the laser beam on the plasma. The energy of the injected particles were tuned to 2.5, 3.0 and 3.3 MeV, to study the effect of the mismatch between the Lorentz factors of the wave and of the particles. The dependence on the light intensity was measured by comparing several laser shots taken through different absorbers. In this article we describe the energy spectra of the accelerated electrons under these different conditions and we interpret them in the frame of a simple model.

\section{Experimental parameters}

This paragraph summarises the experimental parameters relevant to the new data sample. The experimental set-up has already been described in details [6], [8].

Two synchronised laser oscillators, Nd-YLF $(1.0530 \mu \mathrm{m})$ and Nd-YAG $(1.0642 \mu \mathrm{m})$, deliver two pulses of $90 \mathrm{ps}$ and $200 \mathrm{ps}$ duration (FWHM), of $11.8 \mathrm{~J}$ and $4.4 \mathrm{~J}$ energy respectively. After amplification in the LULI laser chain, the beam diameter is 65 $\mathrm{mm}$. The two pulses are synchronised in time and then focused by a $1.5 \mathrm{~m}$ or $1.2 \mathrm{~m}$ focal length lens into the vessel filled with $\mathrm{D}_{2}$ gas. At resonance, the deuterium density is $1.115 \times 10^{17} \mathrm{~cm}^{-3}$ $\pm 2.3 \%$, corresponding to a pressure $\mathrm{P}_{\text {res }}$ equal to $2.272 \mathrm{mb}$ at $22^{0} \mathrm{C}$. It can be adjusted in the vessel with a precision of $\pm 0.3 \%$. The electron source is a pulsed Van de Graaff accelerator delivering electrons with a kinetic energy set to $2.000,2.500$ or 2.800 $\mathrm{MeV}$, i.e. $2.511,3.011$ or $3.311 \mathrm{MeV}$ total energy (in the rest of this article, only total energy will appear). The relative energy fluctuation is $10^{-3}$. The pulse duration is equal to $0.4 \mathrm{~ms}$, so the beam can be considered as continuous during the lifetime of the plasma. The current is set to $200 \mu \mathrm{A}$, which corresponds to 1250 electrons per picosecond. The geometrical parameters have been measured at the plasma level by monitors detecting the optical transition radiation. We found $25 \mu \mathrm{m}$ [RMS] for the focal spot in vacuum, $45 \mu \mathrm{m}$ for the focal spot in $2 \mathrm{mb} \mathrm{D}_{2}$, and $10 \mathrm{mrad}$ [RMS] for the angular divergence.

The energy spectrum of the electrons after passing the plasma is measured by a magnetic spectrograph and an array of 10 scintillators read by photomultipliers tubes. When the injection energy is $3.011 \mathrm{MeV}$ the lowest detectable channel corresponds to electrons between 3.270 and $3.420 \mathrm{MeV}$. The other 9 channels are incremented by steps of $0.154 \mathrm{MeV}$. All these quantities scale proportionally to the injection energy. The angular acceptance for the central channel is $90 \mathrm{mrad}$ horizontally and $110 \mathrm{mrad}$ vertically.

We have found that the noise in the detectors is due mainly to the electrons which scatter on the gas molecules. This noise amounts to about $5 e^{-} / \mathrm{ns}$ for the current of $200 \mu \mathrm{A}$. The photomultiplier signals being gated by $5 \mathrm{~ns}$ long electronic gates, this noise corresponds to $25 e^{-}$per channel. The uncertainty on the electron number is partly due to the calibration process $( \pm 15 \%)$, and partly to the statistical fluctuation of the noise from scattered 

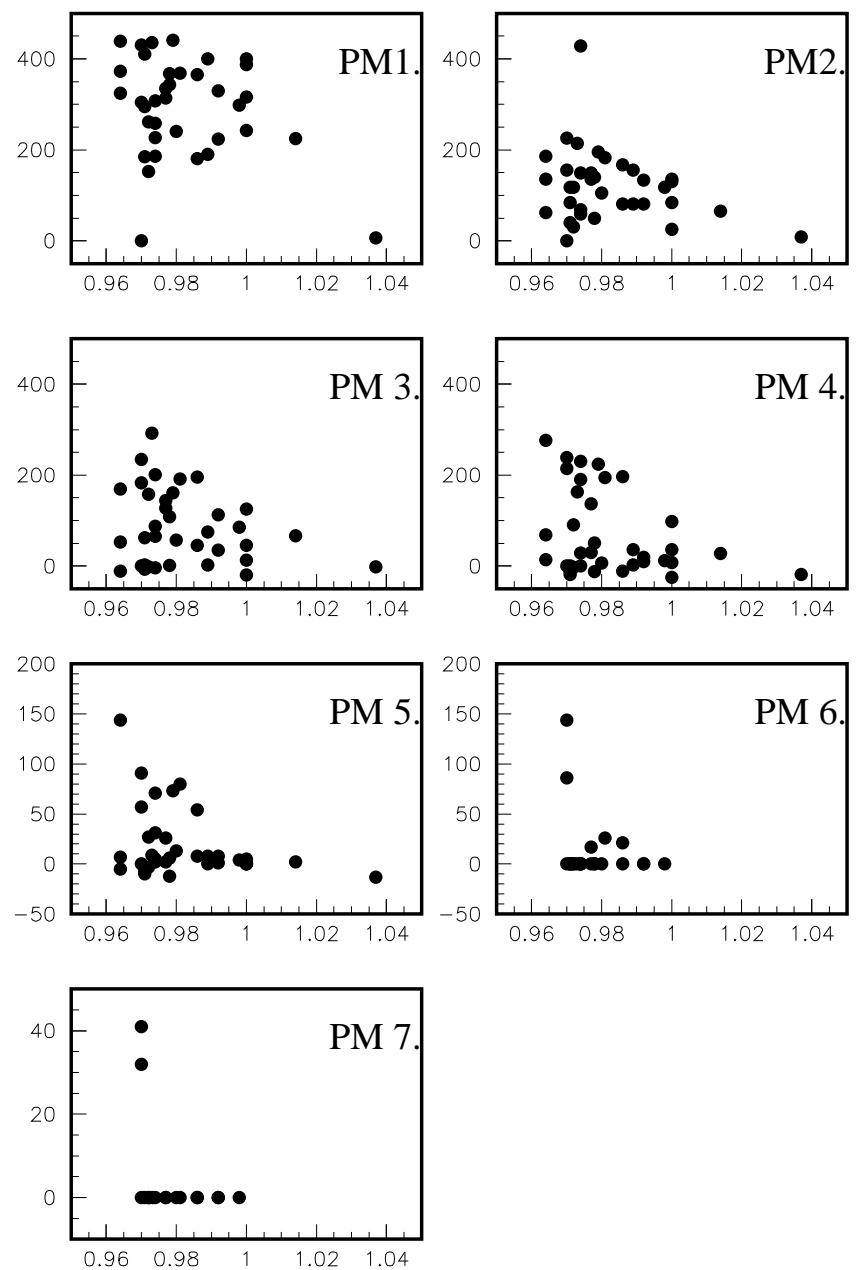

Figure. 1. Number of electrons detected in channel 1 to 7 (3.35 to $4.42 \mathrm{MeV}$ ) as a function of the gas fill pressure normalised to the resonant pressure $\mathrm{P}_{\text {res }}\left(2.272 \mathrm{mbar}\right.$ at $\left.22^{\circ} \mathrm{C}\right)$. The injection energy is $3.01 \mathrm{MeV}$ and the focal length $1.2 \mathrm{~m}$.

electrons $\left( \pm 5 e^{-}\right)$.

The main proof that electrons are accelerated by the beat-wave mechanism is the fact that, as a function of the gas fill pressure, the number of detected electrons exhibits a peak near the resonant pressure $\mathrm{P}_{\text {res }}$. This is evident in figure 1, where we observe also that the width of the resonance peak is narrower for higher energy channels. This can be explained by the high sensitivity of the maximum energy gain to the peak value and to the coherence of the accelerating electric field.

We also performed a number of null tests, consisting of shots with either no electrons, no gas, a single laser wavelength, or two wavelengths separated in time. None of these shots showed a signal of accelerated electrons.

\section{Effect of the injection energy}

The optimum effect of acceleration is expected when the Lorentz factor $\gamma_{p}$ of the plasma wave, determined by its phase velocity, is close to the Lorentz factor $\gamma_{e}$ of the injected electrons. The phase velocity of the plasma wave is equal to the group velocity of the laser beat-wave which, in our case, is fixed by the

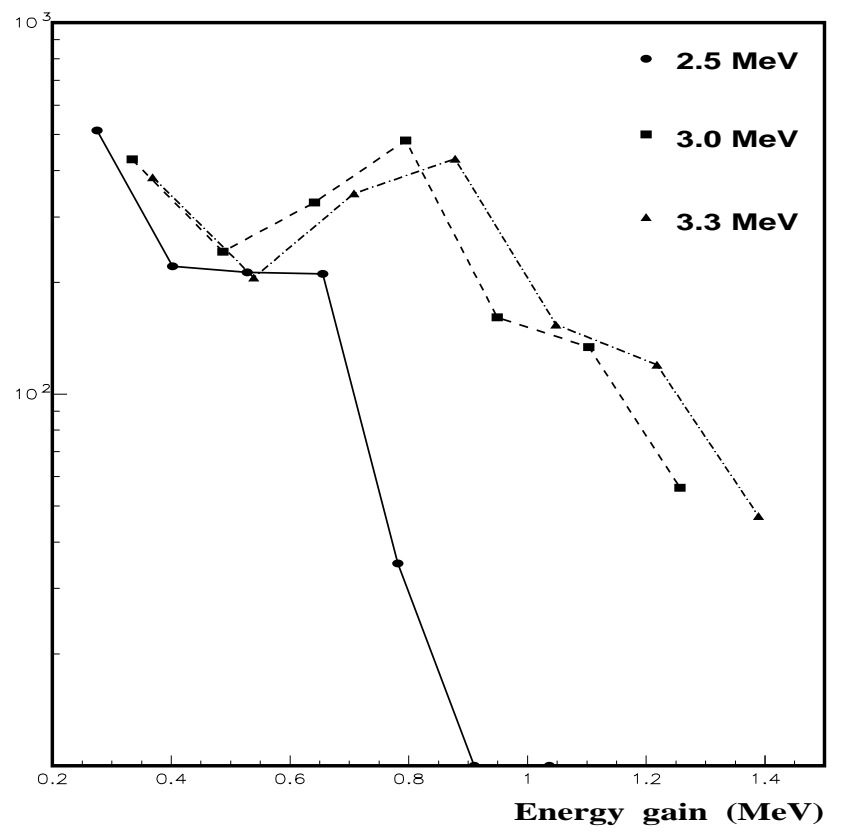

Figure. 2. Number of accelerated electrons, at the resonant pressure, as the function of their energy gain, for three values of the injection energy $\mathrm{E}_{i}$.

Nd-YLF $(1.0530 \mu \mathrm{m})$ and Nd-YAG $(1.0642 \mu \mathrm{m})$ wavelengths. This corresponds to a $\gamma_{p}$ equal to 94.5. The maximum energy of the Van de Graaff injector is $3.3 \mathrm{MeV}$, and the $\gamma_{e}$ is equal to 6.5 , much lower than $\gamma_{p}$. The consequence of this mismatch is that the electrons get out of phase after travelling a distance $l$ in the plasma:

$$
l \approx \gamma_{p} \gamma_{e}^{2} \lambda
$$

where $\lambda$ is the mean laser wavelength. Figure 2 shows the spectra of the accelerated electrons, for the most efficient laser shots, for three different injection energies, with all other parameters being unchanged. The detector channels are not in correspondence because the magnetic field of the spectrometer magnet is different for each injection energy. One can see clearly that the energy gain is correlated with an increase of the injection energy.

\section{Effect of the laser intensity}

Neutral light absorber were positioned in the beam line just upstream of the focusing lens at the entrance of the vessel. The two wavelengths were equally attenuated, successively by factors $0.5,0.29$ and 0.125 . The corresponding spectra of accelerated electrons, accelerated over several shots are shown in figure 3 . The effect is a rapid decrease of the highest energy channels and a complete disappearance of the detected particles with the last absorber.

\section{Effect of the plasma dimension}

We were able to monitor the size of the plasma by recording an image for each laser shot. The diagnostic consisted in a lens placed downstream of the vessel, followed by a CCD camera. The diameter of the image of the focal spot gave the lateral di- 


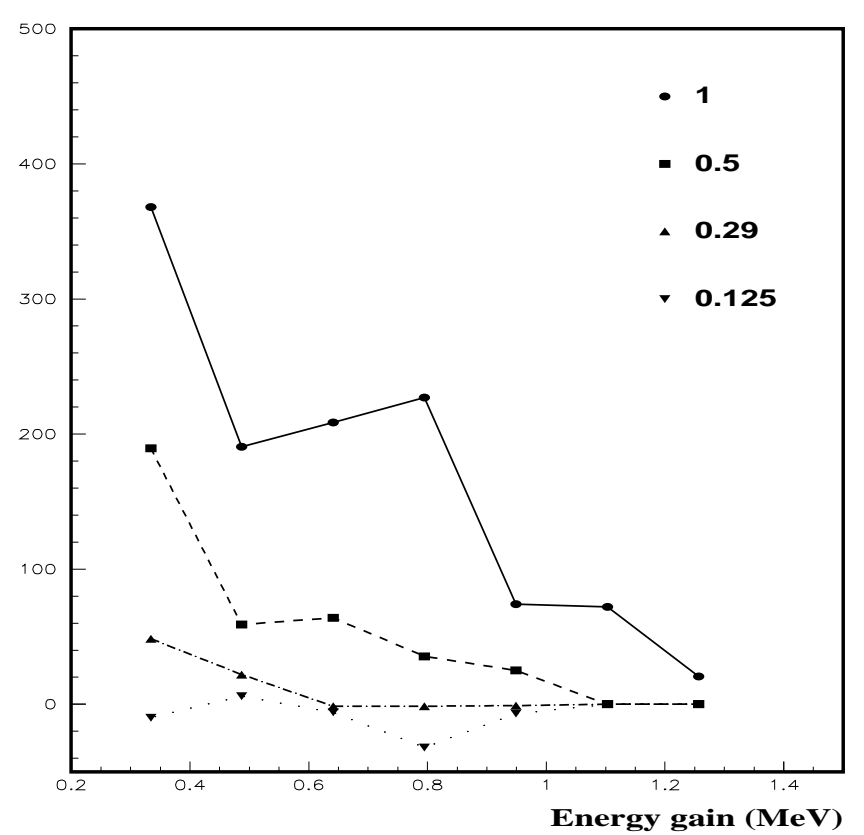

Figure. 3. Number of accelerated electrons, at the resonant pressure, as the function of their energy gain, for four values of the laser relative intensity

mension, whereas the longitudinal size was scanned by moving the diagnostic lens between successive shots.

When we changed the concentration lens focal length from 1.5 $\mathrm{m}$ to $1.2 \mathrm{~m}$, the longitudinal size scaled. On the other hand the lateral size did not change, probably because the $1.2 \mathrm{~m}$ lens has more aberrations. Consequently the variation of the focal length had no effect on the laser intensity in the focal plane.

Figure 4 shows the spectra of accelerated electrons measured with the $1.2 \mathrm{~m}$ and $1.5 \mathrm{~m}$ lenses. The dramatic improvement of the maximum energy can be attributed to the reduced length of the plasma, which is closer to the optimum value (of the order of $3 \mathrm{~mm}$ ) for our $3 \mathrm{MeV}$ injected beam.

\section{Conclusion}

Our new data confirm our previous observation of the first evidence of the acceleration of externally injected electrons by a relativistic plasma wave, excited by beat-waves of a two-frequency Nd:glass laser. The pressure scan shows that the resonance is sharper for the highest energy channels. Nevertheless the optimum conditions are not fulfilled since the Lorentz factor of the injected electrons, equal to 6 , is much smaller than the Lorentz factor of the relativistic plasma wave, equal to 94.5. A clear experimental demonstration of this fact comes from the measurements of the spectra after varying the injection energy or the plasma dimension. A more complete analysis including theoretical simulations is under progress.

\section{Acknowledgments}

We gratefully acknowledge the help of the laser and technical staff of LULI, LPNHE and SESI, and of M. Poitevin, S. Simon and Y. Fournier (DAPNIA). This work was financially supported

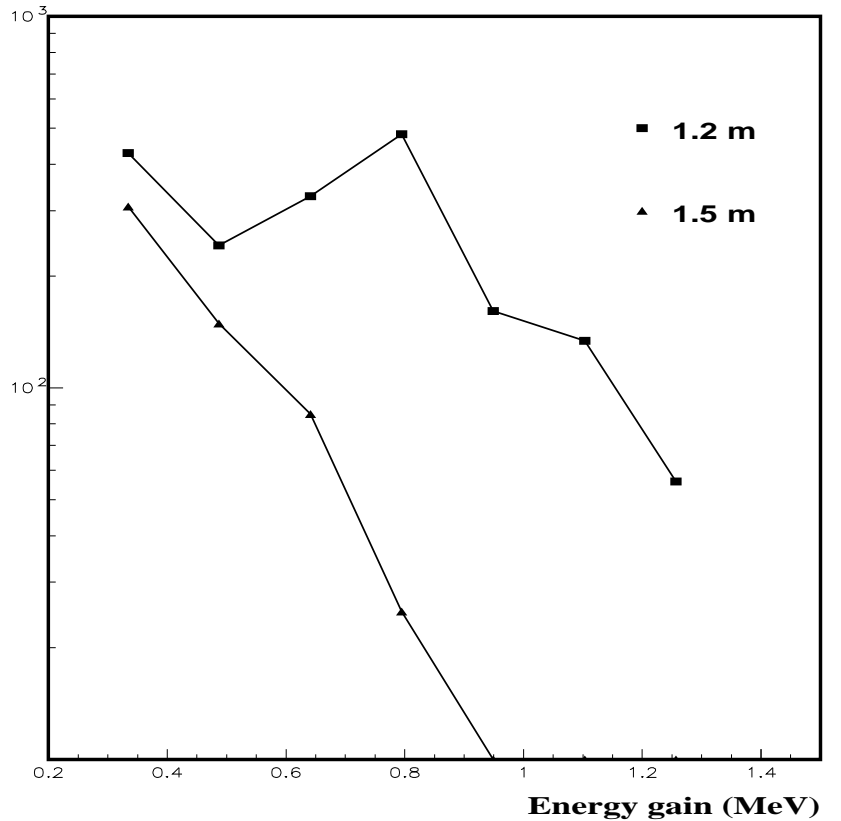

Figure. 4. Number of accelerated electrons, at the resonant pressure, as the function of their energy gain, for two values of the concentrating lens focal length

by Ecole Polytechnique, IN2P3-CNRS, SPI-CNRS, CEA, EEC and DRET.

\section{References}

[1] T. Tajima and J. Dawson, Phys. Rev. Lett. 43, pp. 267-270, 1979.

[2] C.E. Clayton et al., Phys. Plasmas 1, pp. 1753-1759, 1994.

[3] N.A. Ebrahim, J. Appl. Phys. 76, pp. 7645-7647, 1994.

[4] Y. Kitigawa et al., Phys. Rev. Lett. 68, pp. 48-51, 1992.

[5] F. Amiranoff et al., proceeding of the European Particle Accelerator Conference, pp. 787-789, London, 1994.

[6] F. Amiranoff et al., proceedings of the 6th Workshop on Advanced Accelerator Concepts, Lake Geneva, WI, 1994

[7] F. Amiranoff et al., Electron Acceleration in Nd-laser Plasma Beat-Wave Experiments, submitted to Phys. Rev. Lett.

[8] F. Amiranoff et al., The Plasma Beat-Wave Acceleration Experiment at Ecole Polytechnique, Nucl. Instr. and Meth., in press. 